\title{
Special Issue on Thermoelectric Properties of Nanostructured Materials
}

Thermoelectric devices convert thermal energy under a temperature gradient into electrical energy, thus providing a mechanism for sustainable energy harvesting without moving parts [1]. The efficiency of the thermoelectric energy conversion is mainly dependent on the transport properties of thermoelectric materials including electrical conductivity, thermopower (or Seebeck coefficient), and thermal conductivity. The three key properties are adversely correlated in traditional bulk materials, limiting their performance improvement.

Nanostructuring has been found to be effective in alleviating the adverse correlation in thermoelectric transport properties. For example, thermal conductivity variations with 2D and 1D nanostructuring due to phonon scattering are of great interest for suppressing thermal conductivity, which are theoretically and experimentally discussed in this special issue. The substitution of atoms and defects as well as the morphology of nanomaterials can significantly affect both thermal conductivity and electrical properties, which are also included in this issue. Given the extraordinary importance of thermoelectricity from the applied physics viewpoint, this issue focuses on theory and numerical simulation modeling about extraordinary behaviors arising from nanostructuring. At the same time, the most recent advances on low dimensional thermoelectric materials and on methods for the investigation of the thermoelectric properties at the nanoscale are highlighted. The first step for investigating the role of nanostructuring is the characterization of thermoelectric properties, which are often challenging due to the small scale of nanomaterials. And the last step is practical considerations in device operation such as metal contact materials and mechanical properties, which are discussed together here.

This special issue starts with two topical reviews that will likely become reference texts for students approaching the field of thermoelectricity and for trained researchers as well. The first one, by Kim and coworkers, deals with the experimental methods to obtain reliable estimates of the thermoelectric parameters at the nanoscale [2]. In the other Swinkels and Zardo give a detailed account of the role of semiconducting nanowires in both fundamental studies of thermoelectric effect and in emerging thermoelectric devices [3].

Theoretical modeling plays an important role in the design of functional materials with tailor-made properties and thermoelectric materials are no exception. As matter of fact, the landmark papers by Hicks and Dresselhaus [4],[5], where it was shown that low-dimensionality and nanostructuring could be used to decouple the electrical and the thermal conductivity and that are unanimously believed to have opened the field of nanostructured thermoelectrics, are theoretical study.

This Special Issue features six theoretical studies based on numerical atomistic simulations. Semiconducting, Si-based systems are the subject of four of these papers, stressing the importance of these materials because of their low-cost and high impact in current industrial applications. Yet, other promising approaches involving two-dimensional materials and polymers are also explored. 
Zianni [6] studied width-modulated nanowires, focusing on the design of nanoconstrictions that scatter much more strongly phonons than electrons, leading to an enhancement in the ZT figure of merit by a factor of 20-30 with respect to bulk silicon. Termentzidis [7], on the other hand, reviewed a few systems that features a strongly anisotropic thermal transport and discussed the impact that they can have in thermoelectric applications. The cases analyzed are: (i) Si/Ge superlattices, (ii) amorphous/crystalline superlattices, (iii) defected GaN where edge and screw dislocations trigger the thermal anisotropy, and (iv) $\mathrm{Bi}_{2} \mathrm{Te}_{3}$ nanowires grown along different crystallographic orientations.

Alloying has received much attention as a simple route to reduce the thermal conductivity and several reports on SiGe have been published over the latest few years [8],[9],[10],[11],[12]. Lee and Hwang [13], expanding on their previous work on the limit of thermal conductivity on SiGe alloys [14], studied ternary silicongermanium-tin alloys. They showed and quantified to what extent the thermal conductivity of a SiGe alloy can be further reduced by tin doping. Defect scattering is also the topic of the work of Patil et al. [15], who studied the effect of vacancies on the thermopower of $\mathrm{MoS}_{2}$ monolayers.

At variance with the conventional approach to superlattices, where different chemical species are altered in an ordered fashion, Samaraweera et al. [16] tackled the characterization by molecular dynamics of $\mathrm{Si}$ nanotwinned random layer, where the superlattice structure is determined by the periodic introduction of twin defects in an all-Si system.

Genovese et al. [17] centered their attention on poly(3,4-ethylenedioxythiophene), a polymer who is a promising candidate for thermoelectric applications because of its low thermal conductivity. Their main finding was elucidating the role of morphology in the determination of the polymer thermal transport properties. Particularly, they showed how the change from crystalline to nearly amorphous samples results in a more than two orders of magnitude decrease of thermal conductivity. Li et al., on the other hand, studied the mechanical properties, which are to date largely unknown, of lanthanum telluride ( $\left.\mathrm{La}_{3} \mathrm{Te}_{4}\right)$, which is a thermoelectric material with applications in the high temperature range [18]. Finally, Antidormi et al. [19] studied both electrical and thermal transport of twisted graphene nanoribbon in the low temperature ballistic regime, and discussed the potential application in the field of thermoelectricity of these origami-like materials.

Nowadays, cutting edge research is being carried out in the experimental investigation of the thermoelectric properties of nanostructured materials and this Special Issue well reflects these efforts. Recently there have been various attempts to utilize thermoelectric devices to harvest body heat for wearable electronics. To provide the mechanical flexibility for effective heat transfer between human body and thermoelectric devices, two different approaches are introduced here. One approach is to use organic materials particularly conducting polymers such as poly(3,4-ethylenedioxythiophene) [20], and another is to design inorganic materials piecewise flexible [21]. Si-Ge superlattices are the subject of a fruitful collaboration between theory and experiments in the work of Thumfart et al. [22]. 
They measured the cross-plane thermal conductivities of Ge-rich $\mathrm{Si} / \mathrm{Ge}$ superlattices and used the experimental results to obtain parameter-free theoretical estimates of the thermal conductivity by combining first-principles calculations and Boltzmann transport theory.

Device design and optimization are explicitly addressed in the remaining two works of this Special Issue. On the modeling side, Eidelman and coworkers [22] present a model of a thermoelectric generator that uses composite materials obtained by sintering diamond nanoparticles as the main component and that relies on electron drag by ballistic phonons. Chavez et al. [24], on the other hand, present the experimental realization of a thermoelectric generator where the metallization and electrode bonding on the hot side is replaced by a $p-n$ junction between the two semiconductor materials. This approach has the goal of increasing the resistance to thermal-mechanical stress that prevents industrial scale thermoelectric generators to operate with high hot-side temperatures.

[1] Benenti G, Casati G, Saito K, and Whitney R S, 2017 Physics Reports, 694, 1

[2] Kim J, Fleming E, Zhou Y, and Shi L, 2018 J Phys D: Appl. Phys 51103002

[3] Swinkels M Y and Zardo I, 2018 J Phys D: Appl. Phys XX XXXX

[4] Hicks L D and Dresselhaus M S 1993 Phys. Rev. B 4712727

[5] Hicks L D and Dresselhaus M S 1993 Phys. Rev. B 4716631

[6] Zianni X 2018 J Phys D: Appl. Phys 51114003

[7] Termentzidis K 2018 J Phys D: Appl. Phys 51094003

[8] Garg J, Bonini N, Kozinsky B, and Marzari N 2011 Phys. Rev. Lett. 106045901

[9] Amato M, Ossicini S, and Rurali R, 2012 Nano Lett. 122717

[10] Amato M, Palummo M, Rurali R, and Ossicini S 2014 Chem. Rev. 1141371

[11] Yi S and Yu C 2015 J. Appl. Phys. 117035105

[12] Lee E K, Yin L, Lee Y, Lee J W, Lee S J, Lee J, Cha S N, Whang D, Hwang G S, Hippalgaonkar K, Majumdar A, Yu C, Choi B L, Kim J M, and Kim K 2012 Nano Lett. 122918

[13] Lee Y and Hwang G S 2018 J Phys D: Appl. Phys 50494001

[14] Lee Y, Pak A J, and Hwang G S 2016 Phys. Chem. Chem. Phys. 1819544

[15] Patil S B, Sankeshwar N S, and Mulimani B G 2018 J. Phys. D: Appl. Phys. 51 304001

[16] Samaraweera N, Chan K L, and Mithraratne K 2018 J Phys D: Appl. Phys 51 204006

[17] Genovese C, Antidormi A, Dettori R, Caddeo C, Mattoni A, Colombo L, and Melis C 2018 J Phys D: Appl. Phys 50494002

[18] Li G, Aydemir U, Wood M, Goddard III W A, Zhai P, Zhang Q, and Snyder G J 2017 J. Phys. D: Appl. Phys. 50274002

[19] Antidormi A, Royo M, and Rurali R 2018 J Phys D: Appl. Phys 50234005

[20] Galliani D, Battiston S, Ruffo R, Trabattoni S, and Narducci D 2018 J. Phys. D: Appl. Phys. 51034002

[21] Park H, Kim D, Eom Y, Wijethunge D, Hwang J, Kim H, and Kim W 2017 J. Phys. D: Appl. Phys. $\mathbf{5 0} 494006$

[22] Thumfart L, Carrete J, Vermeersch B, Ye N, Truglas T, Feser J, Groiss H, Mingo N and, Rastelli A 2018 J. Phys. D: Appl. Phys. 51014001 
[23] Eidelman E D, Meilakhs A P, Semak B V, and Shakhov F M 2017 J. Phys. D: Appl. Phys. $\mathbf{5 0} 464007$

[24] Chavez R, Angst S, Hall J, Maculewicz F, Stoetzel J, Wiggers H, Hung L T, Van Nong N, Pryds N, Span G, 2018 J. Phys. D: Appl. Phys. 51014005 